\title{
THEORY OF OPERATORS
}

\section{PART I. SINGLE OPERATORS}

\section{F. J. MURRAY}

The work of von Neumann on Operator Theory as distinct from the later work on Rings of operators, extends from 1928 to 1932 with certain later additions. It is a remarkable development in which the inadequacy of formula mathematics for quantum mechanics was established and a new abstract approach introduced. This approach required the abstract definition of Hilbert space and reformulation of the basic Hilbert theory for bounded symmetric operators. Not only was the spectral theory of Hilbert space extended to the unbounded case, for self adjoint operators, but a fascinating new range of phenomena was explored-the symmetric transformations which are not self adjoint. In addition, the foundations for the theory of rings were laid and the canonical resolution which itself was critical in further developments was obtained.

At first glance, the Gram-Schmidt process seems an easy way to replace the notion of an abstract operator by a much more elementary object, an infinite matrix. One simply takes a denumerable set dense in the domain of the operator and orthonormalizes it. The matrix then is obvious and in terms of the matrix we can simply write formulas instead of abstract relations. It certainly is surprising to learn that this matrix does not necessarily give the operator we started out with and yet this possibility must be faced as soon as one tries to specify the operator itself. The operator is determined by certain relations and closure under linearity and in the topological sense. The exact modern form of this relation is in the last paper of this series, i.e., the one on adjoint operators, but the effort to attain such a form is clear throughout. The best examples to indicate the discrepancies between the obvious formulas and the result of closure is given by a sequence of closed symmetric transformatrons, each an extension of the previous one and each with a dense domain. To obtain significant formulas, the abstract structure must be considered; one cannot rely on blind manipulation.

The concept of domain brings to light other critical weaknesses in the obvious formula approach. The quantum mechanics certainly posed the problem of forming the sum or the product of two opera-

Received by the editors February 14, 1958. 
tors. For arbitrary operators the sum of two operators or their product may be trivial because the intersection of their domains is trivial or because the intersection of the range of one with the domain of the other is trivial. The paper on adjoint operations again establishes a significant exception. The product of a transformation with its adjoint always has a significant domain and this fortunately applied to the square of an observable in quantum mechanics.

From the point of view of quantum mechanics, by far the greatest weakness of infinite matrices in the unbounded case, is the fact that their spectral theory simply does not have significance. If a symmetric operator is unbounded above and below it will have infinite matrices bounded one way or the other and this is not a matter of a strange example, but true in the case of every symmetric operator which is unbounded above and below. In the paper on infinite matrices, von Neumann uses this fact to obtain successive choices of axes so that a given matrix eventually corresponds to one for an operator with a pure point spectrum with prescribed values and hence the ultimate equivalence of any two infinite matrices in nine steps. The operators have been altered of course but there is nothing in the choice of coordinates to indicate this.

The extension of the spectral theory from the case of bounded operator to the unbounded case, required the development of an apparatus capable of classifying a wide range of phenomena involving symmetric operators. The self adjoint operators, for which a spectral resolution exists, are a subclass of these. If $A$ is symmetric, the relations $\{(A-i) f,(A+i) f\}$ form the graph of an isometric transformation, $V$. This isometric transformation may be unitary, i.e., it may take the full Hilbert space into itself. For a unitary transformation one can develop the spectral theory. (The bounded self adjoint case is re-established by the use of operational calculus in a fascinating and interesting manner in order to obtain the spectral resolution for unitary transformations.) The Cayley transform permits one to carry this resolution for unitary transformations over to the unbounded self adjoint transformations.

But what if the Cayley transform is not unitary? A whole realm of new possibilities appear. First of all, there is the matter of extending the original transformation. If $V$ maps a proper subspace of Hilbert space into a proper subspace, i.e., if both its range and domain are less than the full space then $V$ can be extended to a more inclusive isometric relation $V^{\prime}$ which in turn is the Cayley transform of a symmetric extension of the original symmetric transformation. The contraction process is beset by the possibility of inadequate domain, 
but the possibilities for extending a symmetric operator are immediately clear from Cayley transform.

But now it is clear that we may have cases in which either the range or domain of the Cayley transform can be the full space but not both. Here, symmetric extension is not possible and yet the given symmetric transformation is not self-adjoint. It is then termed maximal.

The simplest possible example of a symmetric transformation which is not unitary is one for which there exists a complete orthonormal set $\phi_{1}, \phi_{2}, \phi_{3}, \cdots$ such that $V \phi_{n}=\phi_{n+1}$. If the domain of a given $V$ is the full space but the range is not, $V$ must be compounded out of a transformation unitary on subspace and transformations in subspaces each equivalent to the above example. Reversing range and domain, one obtains an analogous result and this in turn permits a complete analysis of the structure in the maximal, symmetric case.

For the non-symmetric closed operator, the canonical resolution as a product of self-adjoint transformation and an isometric transformation indicates completely not only the nature of the range and domain but also the character of the relation between them. The "existence" question is settled here in general, i.e., given $g$ when is there an $f$ such that $g=A f$ ? The canonical resolution is also critical for later development of dimension theory in Rings of operators. But the canonical resolution offers us no hint as to the algebra of a nonsymmetric operator, nor indeed to the whole range of phenomena associated with the Jordan normal form.

The fundamental construct of the adjoint paper is the "graph." Given an operator $A$, the graph of the operator is the set in $\mathfrak{S} \oplus \mathfrak{S}$ consisting of the pairs $\{f, A f\}$. The resulting simplification of the theory is amazing. Notions of closure, domain properties, adjoint properties, all became straight forward geometrical concepts. The essential result, the adequacy of the domain of $A^{*} A$, is the result of a simple projection in $\mathfrak{S} \oplus \mathfrak{S}$. This is the culmination of the geometric point of view for the analysis of operators, which was initiated by the interpretation of characteristic vectors as the principal axes of ellipses.

The paper on The characterization of the spectrum of an integral operator represents the tie-in of the von Neumann operator theory with the integral operator theory. An integral operator is characterized by the fact that zero is the only condensation point of the spectrum.

\section{BIBLIOGRAPHY}

J. von Neumann, Allgemeine Eigenwerttheorie Hermitescher Funktionaloperatoren. Math. Ann. vol. 102 (1929) pp. 49-131. 
$\longrightarrow$ Zur Theorie der unbeschrankten Matrizen, J. Reine Angew Math. vol. 161 (1929) pp. 208-236.

, Über Funktionen von Funktionaloperatoren, Ann. of Math. vol. 32 (1931) pp. 191-226.

$294-310$.

- Characterisierung des Spektuems eines Integraloperators, Actualités Scientifiques et Industrielles, no. 229, Hermann and Cie, Paris, 1935.

Columbia University 